\title{
Elevated Plasma Levels of B-Type Natriuretic Peptide but Not C-Reactive Protein Are Associated With Higher Red Cell Distribution Width in Patients With Coronary Artery Disease
}

\author{
Hidekatsu Fukuta, ${ }^{1}$ MD, Nobuyuki Ohte, ${ }^{1}$ MD, Seiji MukaI, ${ }^{1}$ MD, \\ Tomoaki SAEKI, ${ }^{1}$ MD, Kaoru Asada, ${ }^{1}$ MD, Kazuaki WaKamI, ${ }^{1}$ MD, \\ and Genjiro Kimura, ${ }^{1} \mathrm{MD}$
}

\begin{abstract}
SUMMARY
Although higher red cell distribution width (RDW) has recently been reported to be associated with increased mortality independent of anemia in patients with heart failure and those with coronary artery disease (CAD), the mechanism underlying this association is unknown. We hypothesized that higher RDW may reflect neurohumoral activation and a chronic inflammatory state that each contribute to adverse clinical outcomes in these populations. We measured RDW and plasma levels of B-type natriuretic peptide (BNP) and high-sensitive C-reactive protein (hs-CRP) in 226 consecutive patients undergoing cardiac catheterization for CAD (age, $67 \pm 8$ years; males, 77\%; RDW, $45.8 \pm 3.3 \mathrm{fL}$; hemoglobin, $13.2 \pm 1.4 \mathrm{~g} / \mathrm{dL}$; BNP, median [interquartile range], 26.0 [9.0-58.4] pg/mL; hsCRP, 679 [345-1920] ng/mL).

Plasma BNP $(r=0.21, P<0.01)$ but not hs-CRP $(r=0.04, P>0.1)$ levels correlated with RDW. After adjustment for potential confounders including age, gender, body mass index, glomerular filtration rate, hemoglobin, and known hemodynamic determinants of BNP, including elevated left ventricular end-diastolic pressure and volume and slow left ventricular relaxation, RDW was independently predicted by BNP $\left(r^{2}=0.058, P<0.001\right)$. In conclusion, elevated BNP levels are independently associated with higher RDW in patients with CAD. Neurohumoral activation may be a mechanistic link between increased RDW and adverse clinical outcomes in this population. (Int Heart J 2009; 50: 301-312)
\end{abstract}

Key words: Erythrocyte, Inflammation, Left ventricular hemodynamics, Neurohormones

RED cell distribution width (RDW) is a quantitative measure of the variability in size of circulating erythrocytes. Although this variable is routinely measured by automated hematology analyzers and is reported as part of the complete

From the ${ }^{1}$ Department of Cardio-Renal Medicine and Hypertension, Nagoya City University Graduate School of Medical Sciences, Nagoya, Aichi, Japan.

Address for correspondence: Hidekatsu Fukuta, MD, Department of Cardio-Renal Medicine and Hypertension, Nagoya City University Graduate School of Medical Sciences, 1 Kawasumi Mizuho-cho, Mizuho-ku, Nagoya, Aichi 467-8601, Japan. Received for publication October 9, 2008.

Revised and accepted January 5, 2009. 
blood count, its clinical use is limited to narrowing the differential diagnosis of anemia. $^{1)}$

A retrospective analysis of large clinical datasets has recently shown that higher RDW is associated with increased mortality independent of anemia in patients with heart failure ${ }^{2)}$ and those with coronary artery disease (CAD). ${ }^{3)}$ The mechanism underlying this association, however, is unknown.

Although overtly elevated RDW reflects increased red cell destruction (such as hemolysis), nutritional deficiency (iron, vitamin B12, or folate deficiency), or after blood transfusion, ${ }^{1)}$ patients with these conditions are generally excluded from the studies showing the prognostic association of RDW. ${ }^{2,3)}$ Higher RDW within the normal range may result from ineffective erythropoiesis due to chronic inflammation. Inflammatory cytokines have been found to suppress the maturation of erythrocytes, allowing juvenile erythrocytes entering into circulation and leading to an increase in heterogeneity of the size. ${ }^{4)}$ Furthermore, higher RDW may reflect enhanced erythropoiesis resulting from elevated circulating levels of neurohumoral mediators. Experimental and clinical studies have reported an association between accelerated erythropoiesis and activation of neurohumoral states. ${ }^{5-7)}$ Enhanced erythropoiesis results in an increase in the heterogeneity of circulating erythrocytes. ${ }^{8)}$ Finally, there is increasing evidence that a chronic inflammatory state and neurohumoral activation each contribute to adverse clinical outcomes in patients with $\mathrm{CAD}^{9-12)}$ and those with heart failure. $^{13-16)}$

Accordingly, we hypothesized that inflammatory and neurohumoral activation may be a mechanistic link between higher RDW and increased mortality in patients with CAD. To test our hypothesis, we examined the association of plasma levels of high-sensitive C-reactive protein (hs-CRP) and B-type natriuretic peptide (BNP) with RDW in patients who underwent cardiac catheterization for the evaluation of CAD.

\section{Methods}

Patients: We studied 226 consecutive patients who underwent left-sided cardiac catheterization for the evaluation of CAD and left ventricular hemodynamics and simultaneously had blood drawn for measurements of plasma BNP and hsCRP. All patients had symptoms suggestive of angina and/or clinical signs of $\mathrm{CAD}$, including a positive exercise electrocardiogram, an abnormal myocardial perfusion scintigram, and a previous history of myocardial infarction or coronary revascularization. No patients with acute coronary syndrome, decompensated heart failure, atrial fibrillation, primary valvular diseases, idiopathic dilated or hypertrophic cardiomyopathy, congenital heart disease, renal failure 
(serum creatinine $>2.0 \mathrm{mg} / \mathrm{dL}$ ), severe hepatic diseases, or malignant neoplasms were included. A history of hypertension, diabetes, heart failure, myocardial infarction, and coronary revascularization and medication status were determined by review of medical records. All patients gave written informed consent to participate in the study, and this study was performed according to the regulations proposed by the Ethical Guidelines Committee of the Nagoya City University Graduate School of Medical Sciences.

Cardiac catheterization: Before contrast material was injected into the left ventricle or coronary artery, left ventricular and aortic pressure waves were obtained with a catheter-tipped micromanometer (SPC-454D, Millar Instrument Company, Houston, Texas) and recorded on a polygraph system (RMC-2000, Nihon Kohden, Inc., Tokyo) and on a digital data recorder (NR-2000, Keyence, Osaka, Japan) as reported elsewhere. ${ }^{17-19)}$ From the recorded pressure waves, a time constant of decrease in left ventricular pressure (Tau) was determined by applying a monoexponential fitting with zero asymptote to the left ventricular pressure decay ${ }^{20)}$ Left ventricular end-systolic and end-diastolic volumes were obtained from biplane left ventriculography by use of the method proposed by Chapman, $e t a l^{21)}$ and were used for calculating the ejection fraction. These volumes were corrected for body surface area.

Blood sampling and echocardiography: Venous blood samples for the assay of plasma BNP, hs-CRP, ferritin, and erythropoietin levels were collected from the right femoral vein at cardiac catheterization. Plasma levels of these biomarkers were measured with commercially available kits. Specifically, the BNP levels were measured with a specific immunoradiometric assay for human natriuretic peptides (Shionoria, Shionogi Co., Ltd., Osaka, Japan). The hs-CRP levels were measured by nephrelometric assay (SRL, Tokyo). The ferritin levels were measured using a chemiluminescent enzyme immune assay (SRL). The erythropoietin levels were measured by radioimmuno assay (SRL).

Within a week before the index cardiac catheterization for this study, complete blood count, blood chemistry, and echocardiograms were obtained for assessment of clinical features. Complete blood count was determined using a XE-2100 automated cell counter (Sysmex, Kobe, Japan) with standard calibration. The RDW was taken at the $20 \%$ relation height level, with the red cell histogram peak considered to be $100 \%$. The red cell histogram crosses the $20 \%$ level twice. The distance between the two cross points was defined as RDW and was reported in femtoliters (fL; normal range, 39.0-51.5). The serum creatinine levels were determined by an enzymatic assay method (Kainos CRE-L kit, Kainos, Tokyo). Glomerular filtration rate was estimated by the Cockcroft-Gault formula. ${ }^{22)}$ Left ventricular mass was calculated from M-mode echocardiographic measurements (APLIO 80, Toshiba, Tokyo) ${ }^{23)}$ and was corrected for body sur- 
face area.

Statistical analysis: We used the SAS program package (SAS Institute, Cary, NC) for all statistical analyses. Differences between groups were assessed by Student's $t$-test for normally distributed variables, by the Mann-Whitney $U$ test for non-normally distributed variables, and by $\chi^{2}$ test for categorical variables. The association between continuous variables was determined by Pearson's correlation analysis. Since BNP, hs-CRP, and ferritin values were not normally distributed, a logarithmic transformation was used. To determine the variables independently associated with RDW levels, stepwise multivariate linear regression, including variables that correlated significantly with RDW and those that showed significant differences between patients with higher and lower RDW values, was performed with $P=0.05$ for the threshold for inclusion or exclusion of variables. To further assess the independent association of BNP with RDW, multivariate linear regression was performed by forcefully including gender and known hemodynamic determinants of BNP into the model. $P<0.05$ was considered significant.

\section{Results}

Clinical features: Clinical characteristics of all patients and patient subgroups are shown in Table I. Compared with patients with a below-median $(<44.9 \mathrm{fL})$ RDW value, those with an above-median (> $44.9 \mathrm{fL}$ ) RDW value were more likely to be older and to have a lower body mass index and glomerular filtration rate and higher lactate dehydrogenase and erythropoietin levels. There was no significant difference in serum iron or ferritin levels between the groups. Considering the potential influence of chronic inflammation on serum ferritin levels, we compared ferritin levels adjusted for hs-CRP levels and white blood cell count between groups. There was no significant difference in the adjusted mean levels of ferritin between patients with lower and higher RDW values (adjusted mean $\pm \mathrm{SE}, 4.53 \pm 0.08$ versus $4.56 \pm 0.08 \mathrm{ln}[\mathrm{ng} / \mathrm{mL}], P=\mathrm{NS})$.

The use of medications did not affect RDW or hs-CRP levels. There were no significant differences in RDW levels between patients treated with and without angiotensin converting enzyme inhibitors or angiotensin receptor blockers $(45.6 \pm 3.3$ versus $45.4 \pm 3.2 \mathrm{fL}, P=\mathrm{NS})$ or between patients treated with and without beta blockers $(45.4 \pm 3.2$ versus $45.5 \pm 3.3 \mathrm{fL}, P=\mathrm{NS})$. There were no significant differences in hs-CRP levels between patients treated with and without aspirin (median [interquartile range], 667 [326-1920] versus 765 [404-1880] $\mathrm{ng} / \mathrm{mL}, P=\mathrm{NS})$.

The hemodynamic variables of all patients and patient subgroups are shown in Table I. There were no significant differences in hemodynamic vari- 
Table I. Clinical and Hemodynamic Characteristics of all Patients and Patient Subgroups

\begin{tabular}{|c|c|c|c|c|}
\hline Variable & $\begin{array}{l}\text { All Patients } \\
(n=226)\end{array}$ & $\begin{array}{c}\text { Lower }(<44.9 \\
\text { fL) RDW } \\
(n=111)\end{array}$ & $\begin{array}{l}\text { Higher }(>44.9 \\
\text { fL) RDW } \\
(n=115)\end{array}$ & $\begin{array}{c}P \text { Value, } \\
\text { Lower versus } \\
\text { Higher RDW }\end{array}$ \\
\hline Age, years & $67 \pm 8$ & $66 \pm 8$ & $68 \pm 7$ & $<0.01$ \\
\hline Male gender, $n(\%)$ & $175(77)$ & $82(74)$ & $93(81)$ & NS \\
\hline Body mass index, $\mathrm{kg} / \mathrm{m}^{2}$ & $24.0 \pm 3.2$ & $24.5 \pm 2.9$ & $23.6 \pm 3.4$ & $<0.05$ \\
\hline Hypertension, $n(\%)$ & $113(50)$ & $56(50)$ & $57(50)$ & NS \\
\hline Diabetics, $n(\%)$ & $86(38)$ & $40(36)$ & $46(40)$ & NS \\
\hline History of heart failure, $n(\%)$ & $41(18)$ & $19(17)$ & $22(19)$ & NS \\
\hline Previous myocardial infarction, $n(\%)$ & $129(57)$ & $61(55)$ & $68(59)$ & NS \\
\hline Previous coronary revascularization, $n(\%)$ & $110(49)$ & $60(54)$ & $50(43)$ & NS \\
\hline White blood cell, $10^{3} / \mathrm{mm}^{3}$ & $5.8 \pm 1.7$ & $5.7 \pm 1.5$ & $5.9 \pm 1.8$ & NS \\
\hline Platelet, $10^{3} / \mathrm{mm}^{3}$ & $20.5 \pm 6.3$ & $20.5 \pm 5.2$ & $20.4 \pm 7.2$ & NS \\
\hline RDW, fL & $45.8 \pm 3.3$ & $42.9 \pm 1.5$ & $48.0 \pm 2.5$ & $<0.001$ \\
\hline Serum hemoglobin, $\mathrm{g} / \mathrm{dL}$ & $13.2 \pm 1.4$ & $13.4 \pm 1.2$ & $13.1 \pm 1.5$ & 0.083 \\
\hline Lactate dehydrogenase, U/L & $192 \pm 46$ & $183 \pm 31$ & $201 \pm 56$ & $<0.01$ \\
\hline Ferritin, ng/mL & $102(52.2-174)$ & $88.8(51.4-178)$ & $113(55.1-174)$ & NS \\
\hline Serum iron, $\mu \mathrm{g} / \mathrm{dL} *$ & $98 \pm 39$ & $101 \pm 33$ & $96 \pm 44$ & NS \\
\hline Erythropoietin, $\mathrm{mU} / \mathrm{mL} \dagger$ & $21.2 \pm 8.5$ & $18.8 \pm 6.1$ & $23.3 \pm 9.7$ & $<0.01$ \\
\hline Hs-CRP, ng/mL † & $679(345-1920)$ & $654(285-1420)$ & 705 (402-2400) & NS \\
\hline $\mathrm{BNP}, \mathrm{pg} / \mathrm{mL}$ & $26.0(9.0-58.4)$ & $21.7(8.2-51.6)$ & $29.1(11.1-67.1)$ & 0.07 \\
\hline Serum creatinine, $\mathrm{mg} / \mathrm{dL}$ & $0.84 \pm 0.19$ & $0.83 \pm 0.19$ & $0.85 \pm 0.19$ & NS \\
\hline Glomerular filtration rate, $\mathrm{mL} / \mathrm{min} / 1.73 \mathrm{~m}^{2} \S$ & $77.2 \pm 29.1$ & $81.7 \pm 31.3$ & $73.0 \pm 26.4$ & $<0.05$ \\
\hline LV mass index, $\mathrm{g} / \mathrm{m}^{2}$ & $109.5 \pm 27.4$ & $108.3 \pm 25.3$ & $110.6 \pm 29.4$ & NS \\
\hline Multi-vessel disease, $n(\%)$ ฯ & $106(47)$ & $45(41)$ & $61(53)$ & 0.060 \\
\hline \multicolumn{5}{|l|}{ Medication } \\
\hline ACE-I or ARB, $n(\%)$ & $96(42)$ & $43(39)$ & $53(46)$ & NS \\
\hline$\beta$-Blocker, $n(\%)$ & $96(42)$ & $50(45)$ & $46(40)$ & NS \\
\hline Calcium blocker, $n(\%)$ & $73(32)$ & $34(31)$ & $39(34)$ & NS \\
\hline Diuretics, $n(\%)$ & $43(19)$ & $20(18)$ & $23(20)$ & NS \\
\hline Statins, $n(\%)$ & $130(58)$ & $71(64)$ & $59(51)$ & 0.054 \\
\hline Aspirin, $n(\%)$ & $185(82)$ & $94(84)$ & $91(79)$ & NS \\
\hline Heart rate, beat $/ \mathrm{min}$ & $66 \pm 12$ & $66 \pm 12$ & $66 \pm 11$ & NS \\
\hline LV end-diastolic pressure, $\mathrm{mmHg}$ & $14.6 \pm 5.9$ & $14.8 \pm 6.3$ & $14.4 \pm 5.4$ & NS \\
\hline Mean aortic pressure, $\mathrm{mmHg}$ & $96.8 \pm 15.2$ & $95.4 \pm 13.8$ & $98.1 \pm 16.4$ & NS \\
\hline Tau & $46.5 \pm 9.4$ & $46.6 \pm 9.5$ & $46.4 \pm 9.4$ & NS \\
\hline $\mathrm{LV}$ end-diastolic volume index, $\mathrm{mL} / \mathrm{m}^{2}$ & $85.0 \pm 21.3$ & $85.1 \pm 19.7$ & $85.0 \pm 22.8$ & NS \\
\hline $\mathrm{LV}$ end-systolic volume index, $\mathrm{mL} / \mathrm{m}^{2}$ & $33.5 \pm 18.8$ & $33.2 \pm 17.8$ & $33.9 \pm 19.8$ & NS \\
\hline Ejection fraction & $0.62 \pm 0.13$ & $0.63 \pm 0.13$ & $0.62 \pm 0.14$ & NS \\
\hline
\end{tabular}

Data are expressed as the mean \pm standard deviation, median (interquartile range), or frequency (within-group percentage).

* Serum iron levels were available in 151 patients.

$\dagger$ Serum erythropoietin levels were available in 130 patients.

\$ Normal range: below $1500 \mathrm{ng} / \mathrm{mL}$.

$\S$ Glomerular filtration rate was calculated as follows: $[(140$-age $) \times($ weight $)] / 72 \times$ serum creatinine $(\times 0.85$ if female).

I Diseased coronary artery was defined as a major epicardial artery with $75 \%$ or more stenosis on the angiogram. ACE-I indicates angiotensin converting enzyme inhibitors; ARB, angiotensin receptor blockers; BNP, B-type natriuretic peptide; Hs-CRP, high-sensitivity C-reactive protein; LV, left ventricular; Tau, LV relaxation time constant; and RDW, red cell distribution width. 
Table II. Correlation Coefficients of Clinical and Hemodynamic Variables With Red Cell Distribution Width (RDW)

\begin{tabular}{|c|c|c|}
\hline \multirow{2}{*}{ Variable } & \multicolumn{2}{|c|}{ RDW } \\
\hline & $r$ & $P$ \\
\hline \multicolumn{3}{|l|}{ Clinical variables } \\
\hline Age & 0.20 & $<0.01$ \\
\hline Body mass index & -0.18 & $<0.01$ \\
\hline White blood cell & -0.09 & NS \\
\hline Platelet & -0.04 & NS \\
\hline Hemoglobin & -0.17 & $<0.01$ \\
\hline Lactate dehydrogenase & 0.18 & $<0.01$ \\
\hline Ferritin & -0.001 & NS \\
\hline Serum iron* & -0.08 & NS \\
\hline Erythropoietin $^{\dagger}$ & 0.34 & $<0.01$ \\
\hline Hs-CRP & 0.04 & NS \\
\hline BNP & 0.21 & $<0.01$ \\
\hline Serum creatinine & -0.01 & NS \\
\hline Glomerular filtration rate & -0.10 & NS \\
\hline LV mass index & 0.003 & NS \\
\hline \multicolumn{3}{|l|}{ Hemodynamic variables } \\
\hline Heart rate & -0.03 & NS \\
\hline LV end-diastolic pressure & 0.03 & NS \\
\hline Mean aortic pressure & 0.03 & NS \\
\hline Tau & 0.03 & NS \\
\hline LV end-diastolic volume index & -0.01 & NS \\
\hline LV end-systolic volume index & 0.04 & NS \\
\hline Ejection fraction & -0.08 & NS \\
\hline
\end{tabular}

Abbreviations as in Table I.

* Serum iron levels were available in 151 patients.

${ }^{\dagger}$ Serum erythropoietin levels were available in 130 patients.

ables between patients with below-median RDW values and those with abovemedian RDW values.

Correlation of clinical and hemodynamic variables with RDW: The correlation coefficients of clinical and hemodynamic variables with RDW are shown in Table II. Plasma BNP but not hs-CRP levels correlated significantly with RDW (Figure). When analyses were performed separately for males and females, BNP levels correlated significantly with RDW (correlation coefficients for males and females, 0.17 and $0.39, P<0.05$ and $P<0.01$, respectively). In stepwise multivariate linear regression including age, body mass index, glomerular filtration rate, lactate dehydrogenase, and hemoglobin, RDW was predicted by BNP levels $\left(r^{2}=0.047, P<0.01\right)$ and age $\left(r^{2}=0.032, P<0.01\right.$; Table III). Even when gender and known hemodynamic determinants of BNP levels, including left ventricular end-diastolic pressure and volume and a time constant of decrease in left ventricular pressure (Tau) were forcefully entered into the model, RDW was independently predicted by BNP levels $\left(r^{2}=0.058, P<0.001\right)$. Erythropoietin 


$$
r=0.21, P<0.01
$$

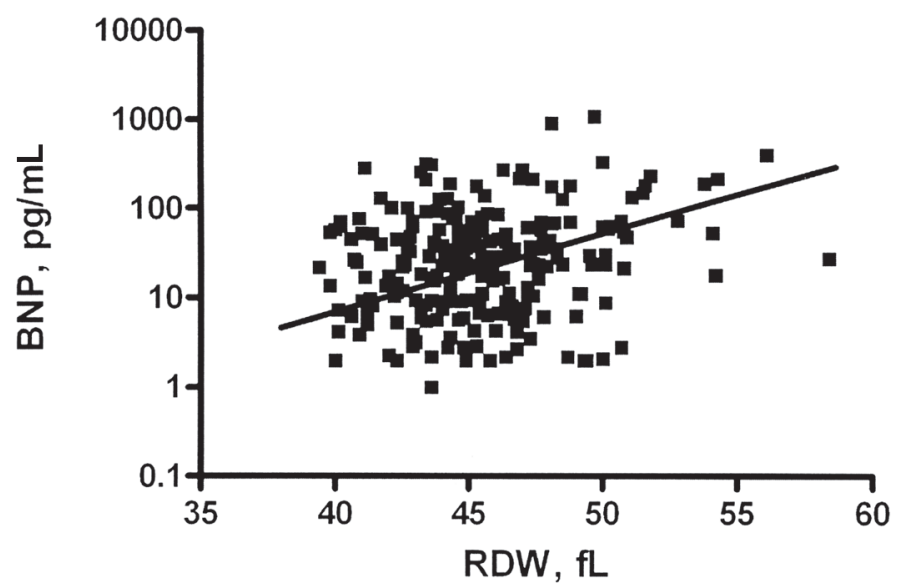

$$
r=0.04, P>0.1
$$

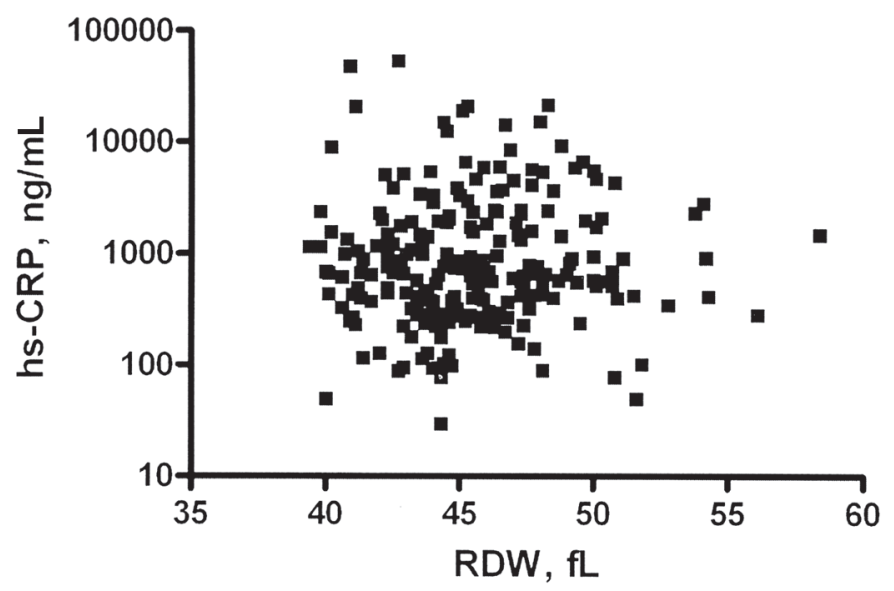

Figure. Correlation between plasma B-type natriuretic peptide (BNP) and high-sensitive C-reactive protein (hs-CRP) levels and red cell distribution width (RDW).

Table III. Stepwise Multivariate Linear Regression for Red Cell Distribution Width

\begin{tabular}{lcccc}
\hline \multicolumn{1}{c}{ Variable } & $\beta$ Coefficient & Standard Error & Partial $r^{2}$ & $P$ Value \\
\hline BNP, $\ln (\mathrm{pg} / \mathrm{mL})$ & 0.4773 & 0.1609 & 0.047 & $<0.01$ \\
Age, years & 0.0730 & 0.0265 & 0.032 & $<0.01$ \\
\hline
\end{tabular}

Ln indicates logarithmic transformation. Other abbreviations as in Table I. 
levels were available in 130 patients. Erythropoietin levels correlated significantly with RDW ( $r=0.34, P<0.01)$ but not with BNP levels $(r=-0.028, P=$ NS). When erythropoietin was forcefully entered into the multivariate model listed in Table III, RDW was predicted by BNP $\left(r^{2}=0.039, P<0.05\right)$ and erythropoietin $\left(r^{2}=0.116, P<0.001\right)$ levels.

\section{Discussion}

Major findings: In the present study, we hypothesized that a chronic inflammatory state and neurohumoral activation may be a mechanistic link between higher RDW and increased mortality in patients with CAD. To test our hypothesis, we examined the association of hs-CRP and BNP levels with RDW in patients undergoing cardiac catheterization for the evaluation of CAD. We observed that elevated BNP but not hs-CRP levels were associated with higher RDW and that the association of BNP levels with RDW was independent of potential confounders, including age, gender, renal function, body mass index, and hemoglobin levels, and also of known hemodynamic determinants of BNP levels including elevated left ventricular end-diastolic pressure and volume and slow left ventricular relaxation. Thus, we found that elevated BNP but not hs-CRP levels are independently associated with higher RDW in patients with CAD. These results indicate that neurohumoral activation but not a chronic inflammatory state may partially mediate the association between higher RDW and increased mortality in patients with CAD.

Comparison with earlier studies: Two recent studies have shown that higher RDW is associated with increased mortality in patients with heart failure and those with CAD. ${ }^{2,3)}$ Specifically, in the CHARM (Candesartan in Heart Failure: Assessment of Reduction in Mortality and Morbidity) trial including 2679 heart failure patients, among routine biochemical and hematologic measures, higher RDW showed the greatest association with morbidity and mortality (adjusted hazard ratio, 1.17 per 1 -SD increase, $P<0.001) .{ }^{2)}$ Similarly, a graded independent relation between higher levels of RDW and the risk of death and cardiovascular events was found in the CARE (Cholesterol and Recurrent Events) trial that included 4111 stable CAD patients without heart failure; patients with RDW in the highest quartile had an adjusted hazard ratio for death of 1.78 (95\% CI, 1.28-2.47), compared with those in the lowest quartile." Although these studies reported clinical variables associated with higher RDW, including age, reduced body mass index, anemia, and renal dysfunction, they did not examine neurohumoral and inflammatory correlates of RDW. The present study confirms clinical correlates of RDW found in earlier studies ${ }^{3)}$ and further demonstrates that elevated BNP but not hs-CRP levels were associated with RDW in CAD 
patients independent of potential confounders including known hemodynamic determinants of elevated BNP levels.

Possible mechanisms: When considering possible mechanisms for our observations, it may be useful to look over the pathophysiological background of higher RDW and BNP levels. RDW is a quantitative measure of the variability in size of circulating erythrocytes and higher values reflect a more heterogeneous cell population. Although overtly elevated RDW reflects increased red cell destruction, nutritional deficiency, or after blood transfusion, ${ }^{1)}$ most of our patients had normal RDW values. Thus, higher RDW in the present study may be due either to (1) ineffective erythropoiesis resulting from chronic inflammation ${ }^{4)}$ or to (2) enhanced erythropoiesis stimulated by increased production of erythropoietin. ${ }^{8,24}$ Our observations that elevated levels of erythropoietin but not hs-CRP were associated with higher RDW indicate that the latter is a likely cause of higher RDW in our patients. Although BNP is produced mainly by the ventricle in response to an increase in wall stretch and exerts natriuretic and vasodilatory properties, a variety of vasoconstrictive neurohormones including angiotensin II and norepinephrine have been found to directly enhance BNP secretion. ${ }^{25)}$ Furthermore, these neurohormones have been reported to stimulate erythropoiesis by increasing erythropoietin production. ${ }^{6,7)}$ From these aspects, enhanced erythropoiesis due probably to activated renin-angiotensin and sympathetic nervous systems may explain our observed association of elevated BNP levels with higher RDW. Thus, higher RDW in CAD patients may reflect the presence of neurohumoral and endocrine activation.

In the present study, however, the use of beta-blockers, angiotensin converting enzyme inhibitors, or angiotensin receptor blockers was not associated with lower RDW. Nevertheless, given the cross-sectional nature of the present study, prospective studies are warranted to examine the effect of neurohumoral blockade on RDW in CAD patients with higher RDW.

Although chronic inflammation has been hypothesized as a possible mechanism underlying the association between higher RDW and increased mortality in cardiac patients, ${ }^{2,3)}$ the present study is unlikely to support the hypothesis, at least in patients with stable CAD. Nevertheless, the contribution of pro-inflammatory cytokines, including tumor necrosis factor and interleukin-6, as well as oxidative stress, to higher RDW in patients with CAD and those with heart failure merits further investigation.

Consistent with earlier studies, ${ }^{3)}$ we observed that age was associated with increased RDW. Furthermore, the association was independent of potential confounders including renal function and serum hemoglobin and erythropoietin levels, suggesting the direct impact of age on erythropoietic activity.

Limitations: There are several limitations in the present study. First, our study 
patients were composed of stable CAD patients without overt heart failure symptoms. Our findings cannot be generalized to other populations. Second, although the correlation between BNP and RDW was statistically significant, the variability in RDW explained by the variability of BNP was modest. It should be emphasized that plasma BNP level is only one out of many determinants of RDW in CAD patients. Furthermore, a direct cause-effect relation could not be verified because of the cross-sectional nature of the present study. Third, although we speculate that the association of elevated BNP levels with higher RDW in CAD patients may result from enhanced erythropoiesis due to activated renin-angiotensin and sympathetic nervous systems, we did not measure plasma levels of angiotensin II or norepinephrine in our patients. Fourth, reticulocyte count and vitamin B12 and folate levels were not measured. However, our observations that only a small fraction of patients $(n=27$ [12\%]) had increased ( $>100 \mathrm{fL}$ ) red cell mean corpuscular volume and that all of these patients had a red cell mean corpuscular volume of $110 \mathrm{fL}$ or less make it unlikely that deficiency of these vitamins was a major cause of increased RDW in our patients. Finally, we do not have data on partial pressure of arterial oxygen or arterial oxygen saturation in our patients. Hypoxia stimulates erythropoietin production in the kidneys and increases its circulating plasma levels, resulting in enhanced erythroid proliferation in the bone marrow. The hypoxia-induced activated erythropoiesis may increase the heterogeneity of circulating erythrocytes, thereby increasing RDW. Furthermore, hypoxia may directly stimulate BNP secretion in cardiac myocytes. ${ }^{26)}$ Thus, it is possible that our observed association of elevated BNP levels with increased RDW may be mediated by hypoxia. However, all of our patients were in stable condition and patients with decompensated heart failure were not included in the present study, making the possibility less likely.

Clinical implications: Given that elevated RDW and BNP levels are both predictive of adverse clinical outcomes in CAD patients, our findings may have implications for patient evaluation. Studies have shown that elevated plasma levels of BNP and N-terminal pro-BNP are associated with adverse prognosis in CAD patients. ${ }^{9-12)}$ Routine or serial BNP measurements in these patients, however, are hampered by its cost. In contrast, RDW is routinely reported as part of the complete blood count and its measurement requires no additional cost. Our findings suggest that RDW measurement in CAD patients may identify a high-risk subgroup that warrants evaluation of neurohumoral status.

\section{REFERENCES}

1. Evans TC, Jehle D. The red blood cell distribution width. J Emerg Med 1991; 9 Suppl 1: 71-4. (Review)

2. Felker GM, Allen LA, Pocock SJ, et al. Red cell distribution width as a novel prognostic marker in 
heart failure: data from the CHARM Program and the Duke Databank. J Am Coll Cardiol 2007; 50: 40-7.

3. Tonelli M, Sacks F, Arnold M, Moye L, Davis B, Pfeffer M: for the Cholesterol and Recurrent Events (CARE) Trial Investigators. Relation Between Red Blood Cell Distribution Width and Cardiovascular Event Rate in People With Coronary Disease. Circulation 2008; 117: 163-8.

4. Pierce CN, Larson DF. Inflammatory cytokine inhibition of erythropoiesis in patients implanted with a mechanical circulatory assist device. Perfusion 2005; 20: 83-90.

5. Kato H, Ishida J, Imagawa S, et al. Enhanced erythropoiesis mediated by activation of the reninangiotensin system via angiotensin II type 1a receptor. FASEB J 2005; 19: 2023-5.

6. Vlahakos DV, Kosmas EN, Dimopoulou I, et al. Association between activation of the renin-angiotensin system and secondary erythrocytosis in patients with chronic obstructive pulmonary disease. Am J Med 1999; 106: 158-64.

7. Biaggioni I, Robertson D, Krantz S, Jones M, Haile V. The anemia of primary autonomic failure and its reversal with recombinant erythropoietin. Ann Intern Med 1994; 121: 181-6.

8. Sowade O, Sowade B, Gross J, et al. Evaluation of erythropoietic activity on the basis of the red cell and reticulocyte distribution widths during epoetin beta therapy in patients undergoing cardiac surgery. Acta Haematol 1998; 99: 1-7.

9. Ndrepepa G, Kastrati A, Braun S, et al. N-terminal probrain natriuretic peptide and C-reactive protein in stable coronary heart disease. Am J Med 2006; 119: 355e1-8.

10. Omland T, Richards AM, Wergeland R, Vik-Mo H. B-type natriuretic peptide and long-term survival in patients with stable coronary artery disease. Am J Cardiol 2005; 95: 24-8.

11. Kragelund C, Grønning B, Køber L, Hildebrandt P, Steffensen R. N-terminal pro-B-type natriuretic peptide and long-term mortality in stable coronary heart disease. N Engl J Med 2005; 352: 666-75.

12. Dai DF, Hwang JJ, Lin JL, et al. Joint effects of N-terminal pro-B-type-natriuretic peptide and C-reactive protein vs angiographic severity in predicting major adverse cardiovascular events and clinical restenosis after coronary angioplasty in patients with stable coronary artery disease. Circ J 2008; 72 : 1316-23.

13. George J, Patal S, Wexler D, et al. Circulating erythropoietin levels and prognosis in patients with congestive heart failure: comparison with neurohormonal and inflammatory markers. Arch Intern Med 2005; 165: 1304-9.

14. Ishikawa C, Tsutamoto T, Fujii M, Sakai H, Tanaka T, Horie M. Prediction of mortality by high-sensitivity C-reactive protein and brain natriuretic peptide in patients with dilated cardiomyopathy. Circ J 2006; 70: 857-63.

15. Tsutamoto T, Sakai H, Nishiyama K, et al. Direct comparison of transcardiac increase in brain natriuretic peptide (BNP) and N-terminal proBNP and prognosis in patients with chronic heart failure. Circ J 2007; 71: 1873-8.

16. Nishio $\mathrm{Y}$, Sato $\mathrm{Y}$, Taniguchi R, et al. Cardiac troponin $\mathrm{T}$ vs other biochemical markers in patients with congestive heart failure. Circ J 2007; 71: 631-5.

17. Goto T, Ohte N, Miyabe H, et al. Usefulness of plasma brain-type natriuretic peptide level to differentiate left ventricular diastolic dysfunction from preserved diastolic function in patients with systolic dysfunction. Am J Cardiol 2005; 95: 1383-5.

18. Yoshida T, Ohte N, Narita H, et al. Lack of inertia force of late systolic aortic flow is a cause of left ventricular isolated diastolic dysfunction in patients with coronary artery disease. J Am Coll Cardiol 2006; 48: 983-91.

19. Fukuta H, Ohte N, Mukai S, Saeki T, Kobayashi K, Kimura G. Anemia is an independent predictor for elevated plasma levels of natriuretic peptides in patients undergoing cardiac catheterization for coronary artery disease. Circ J 2008; 72: 212-7.

20. Weiss JL, Frederiksen JW, Weisfeldt ML. Hemodynamic determinants of the time-course of fall in canine left ventricular pressure. J Clin Invest 1976; 58: 751-60.

21. Chapman CB, Baker O, Reynolds J, Bonte FJ. Use of biplane cinefluorography for measurement of ventricular volume. Circulation 1958; 18: 1105-17.

22. Cockcroft DW, Gault MH. Prediction of creatinine clearance from serum creatinine. Nephron 1976; 
16: $31-41$.

23. Schiller NB, Shah PM, Crawford M, et al. Recommendations for quantitation of the left ventricle by two-dimensional echocardiography. American Society of Echocardiography Committee on Standards, Subcommittee on Quantitation of Two-Dimensional Echocardiograms. J Am Soc Echocardiogr 1989; 2: 358-67. (Review)

24. Kario K, Matsuo T, Nakao K, Yamaguchi N. The correlation between red cell distribution width and serum erythropoietin titres. Clin Lab Haematol 1991; 13: 222-3.

25. Ruskoaho H. Cardiac hormones as diagnostic tools in heart failure. Endocr Rev 2003; 24: 341-56. (Review)

26. Hopkins WE, Chen Z, Fukagawa NK, Hall C, Knot HJ, LeWinter MM. Increased atrial and brain natriuretic peptides in adults with cyanotic congenital heart disease: enhanced understanding of the relationship between hypoxia and natriuretic peptide secretion. Circulation 2004; 109: 2872-7. 